\title{
Observation of an Inverse Energy Cascade in Developed Acoustic Turbulence in Superfluid Helium
}

\author{
A. N. Ganshin, ${ }^{1}$ V. B. Efimov, ${ }^{1,2}$ G. V. Kolmakov, ${ }^{2,1, *}$ L. P. Mezhov-Deglin, ${ }^{2}$ and P. V. E. McClintock ${ }^{1}$ \\ ${ }^{1}$ Department of Physics, Lancaster University, Lancaster, LA1 4YB, United Kingdom \\ ${ }^{2}$ Institute of Solid State Physics RAS, Chernogolovka, Moscow region, 142432, Russia
}

(Received 20 May 2008; published 8 August 2008)

\begin{abstract}
We report observation of an inverse energy cascade in second sound acoustic turbulence in He II. Its onset occurs above a critical driving energy and it is accompanied by giant waves that constitute an acoustic analogue of the rogue waves that occasionally appear on the surface of the ocean. The theory of the phenomenon is developed and shown to be in good agreement with the experiments.
\end{abstract}

PACS numbers: 67.25.dk, 47.20.Ky, 47.27.-i, 67.25.dt

A highly excited state of a system with numerous degrees of freedom, characterized by a directional energy flux through frequency scales, is referred to as turbulent $[1,2]$. Like the familiar manifestations of vortex turbulence in fluids, turbulence can also occur in systems of waves, e.g., turbulence of sound waves in oceanic waveguides [3], magnetic turbulence in interstellar gases [4], shock waves in the solar wind and their coupling with Earth's magnetosphere [5], and phonon turbulence in solids [6]. Following the ideas of Kolmogorov, the universally accepted picture says that nonlinear wave interactions give rise to a cascade of wave energy towards shorter and shorter wavelengths until, eventually, it becomes possible for viscosity to dissipate the energy as heat. Experiments and calculations show that, most of the time, the Kolmogorov picture is correct $[2,7,8]$.

We demonstrate below that this picture is incomplete. Our experiments with second sound (temperature-entropy) waves in He II show that, contrary to the conventional wisdom, acoustic wave energy can sometimes flow in the opposite direction too. We note that inverse energy cascades are known in 2-dimensional incompressible liquids and Bose gases [9], and have been considered for quantized vortices [10].

We find that energy backflow in our acoustic system is attributable to a decay instability (cf. the kinetic instability in turbulent systems [11]), controlled mainly by nonlinear decay of the wave into two waves of lower frequency governed by the energy (frequency) conservation law [2] $\omega_{1}=\omega_{2}+\omega_{3}$. Here $\omega_{i}=u_{20} k_{i}$ is the frequency of a linear wave of wave vector $k_{i}$ and $u_{20}$ is the second sound velocity at negligibly small amplitude. The instability manifests itself in the generation of subharmonics. A quite similar parametric process, due to 4-wave scattering (modulation instability), is thought to be responsible for the generation of large wind-driven ocean waves [12]. Decay instabilities (especially threshold and nearthreshold behavior) have been studied for, e.g., spin waves [13], magnetohydrodynamic waves in plasma [14], and interacting first and second sound waves in superfluid helium near the superfluid transition [15].
We now discuss what happens to a system of acoustic waves far beyond the decay threshold. Modeling the resultant nonlinear wave transformations in the laboratory is a potentially fruitful approach that has already yielded important results for, e.g., the turbulent decay of capillary waves on the surface of liquid $\mathrm{H}_{2}$ [16]. Here, we exploit the special properties of second sound [17], which enable fundamental wave processes to be studied under laboratory conditions. Its velocity $u_{2}$ depends strongly on its amplitude $\delta T$ and can be approximated as

$$
u_{2}=u_{20}(1+\alpha \delta T) \text {, }
$$

where the nonlinearity coefficient $\alpha$ [18], which determines the strength of the wave interactions, can be made large if the temperature is set right.

Our experiments make use of the high $Q$ cryoacoustical resonator described previously [7], excited close to one of its resonant frequencies. It enables very large second sound standing wave amplitudes to be attained for modest levels of excitation. Its $Q$ factor, determined from the widths of longitudinal resonances at small heat flux densities, was $Q \sim 1000$ for resonant numbers $p \leq 10$ and $Q \sim 3000$ for $30<p<100$. Figure 1 presents typical results obtained when driving at a relatively high resonant frequency $\omega_{d}$ (the 96th longitudinal resonance of the cell). Those in Figs. 1(a) and 1(b) reproduce our earlier observation of the direct Kolmogorov-like cascade of second sound waves in He II [7], when driving on resonance. Figures 1(c) and 1(d) show that tiny changes in driving frequency can produce marked changes in the shape and spectrum of the standing wave. The formation of the spectral peaks near $\frac{1}{2}, \frac{1}{3}$, and $\frac{2}{3} \times \omega_{d}$ satisfies the frequency conservation law with $\omega_{1}=\omega_{d}$, supporting our inference that the instability is controlled mainly by 3 -wave interactions. When the instability develops, huge distortions of the initially periodic signal occur. Although it remains nearly periodic, its characteristic period exceeds that of the driving force and its amplitude can become more than twice that at the driving frequency.

To characterize the instability quantitatively, we use the energy contained in the low-frequency part of the spectrum 

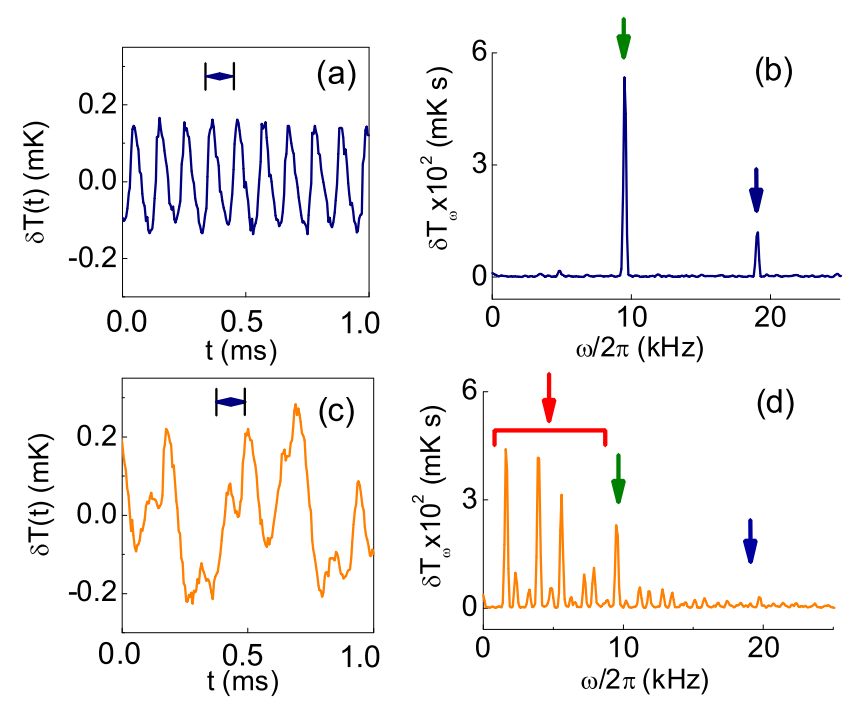

FIG. 1 (color online). Evolution of the observed wave shape in the resonator (left column) and of the power spectrum of second sound standing waves (right) with increasing drive frequency $\omega_{d}$ near the 96th resonance: $\omega_{d} / 2 \pi=9530.8 \mathrm{~Hz}$ (a),(b) and $9535.2 \mathrm{~Hz}$ (c),(d). The ac heat flux density was $W=$ $42 \mathrm{~mW} / \mathrm{cm}^{2}$. The temperature $T=2.08 \mathrm{~K}$ corresponded to negative nonlinearity. The fundamental and first harmonic in (b),(d) are indicated by vertical (green and blue) arrows at $\sim 10$ and $20 \mathrm{kHz}$; the low-frequency domain where the subharmonics appear is indicated by the horizontal range in (d) under the leftmost vertical (red) arrows. The horizontal arrows in (a),(c) indicate the fundamental period of a wave at the driving frequency.

$\omega<\omega_{d}$,

$$
E_{\mathrm{LF}}=\frac{1}{2}\left(\frac{\partial C}{\partial T}\right) \sum_{\omega<\omega_{d}}\left|\delta T_{\omega}\right|^{2}
$$

as an indicator. Figure 2 shows the dependence of $E_{\mathrm{LF}}$ on the ac heat flux density $W$, when driving on the 96th resonance close to $2.08 \mathrm{~K}$. For small $W$ we did not observe any subharmonic generation at all [7]. Then, above a critical flux $W_{c}, E_{\mathrm{LF}}$ rose rapidly, suggesting that the phenomenon is of a threshold character. At $T=T_{\alpha}=$ $1.88 \mathrm{~K}$ for which $\alpha$ vanishes [18], no subharmonics were observed, regardless of the magnitude of $W$, thus confirming the crucial importance of nonlinearity. For $W$ above $10.4 \mathrm{~mW} / \mathrm{cm}^{2}$, we observed a distortion of the signal similar to that shown in Fig. 1(c) and the formation of a few subharmonics. Further increase of $W$ above $20 \mathrm{~mW} / \mathrm{cm}^{2}$ led to the generation of multiple subharmonics. These phenomena appear in the regime where the energy cascade towards the high-frequency domain (i.e., direct cascade, with a Kolmogorov-like spectrum $[7,8])$ is already well developed; see also Fig. 4.

All the above results correspond to steady-state regimes of the wave system. In Fig. 3 we illustrate the transient processes observed after a steplike shift of the driving

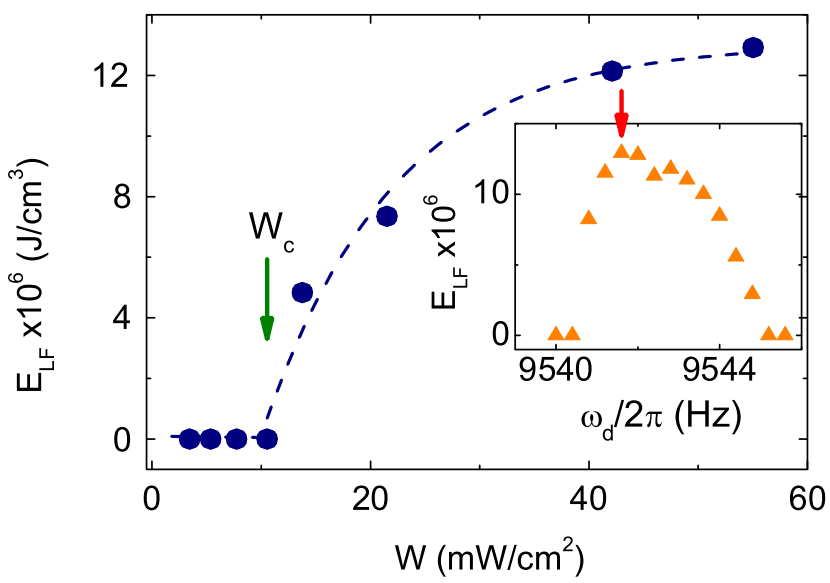

FIG. 2 (color online). The energy $E_{\mathrm{LF}}$ contained in the lowfrequency part of the spectrum as a function of the ac heat flux density $W$, while driving near to the 96th resonance for $T \simeq$ $2.08 \mathrm{~K}$. The threshold value of $W$, marked by the (green) arrow, was $W_{c}=10.4 \mathrm{~mW} / \mathrm{cm}^{2}$. The points are from experiment; dashed lines are guides to the eye. Inset: The dependence of $E_{\mathrm{LF}}$ on $\omega_{d}$, measured for $W=55.6 \mathrm{~mW} / \mathrm{cm}^{2}$; the (red) arrow labels the maximum value of $E_{\mathrm{LF}}$, which is taken to the main figure.

frequency from a frequency initially set far from any resonance to the 96th resonance frequency for $W=$ $42.1 \mathrm{~mW} / \mathrm{cm}^{2}, T=2.08 \mathrm{~K}$. We find that harmonics of the drive in the high-frequency spectral domain are formed very quickly, but that formation of the subharmonics takes much longer: it took $\sim 0.5 \mathrm{~s}$ here, and can reach several tens of seconds under some conditions [19]. It is evident from the inset in Fig. 3 that, as the instability develops, isolated "rogue waves" appear in the signal. As time

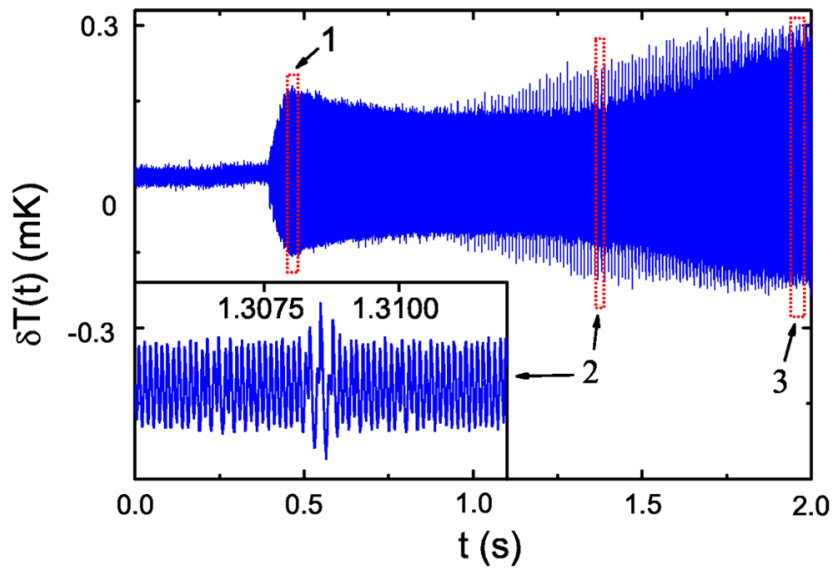

FIG. 3 (color online). Transient evolution of the 2nd sound wave amplitude $\delta T$ after a steplike shift of the driving frequency to the 96th resonance at time $t=0.397 \mathrm{~s}$. Signals in frames 1 and 3 are similar to those obtained in steady-state measurements, Figs. 1(a) and 1(c), respectively. Formation of isolated "rogue" waves is clearly evident. Inset: Example of a rogue wave, enlarged from frame 2 . 


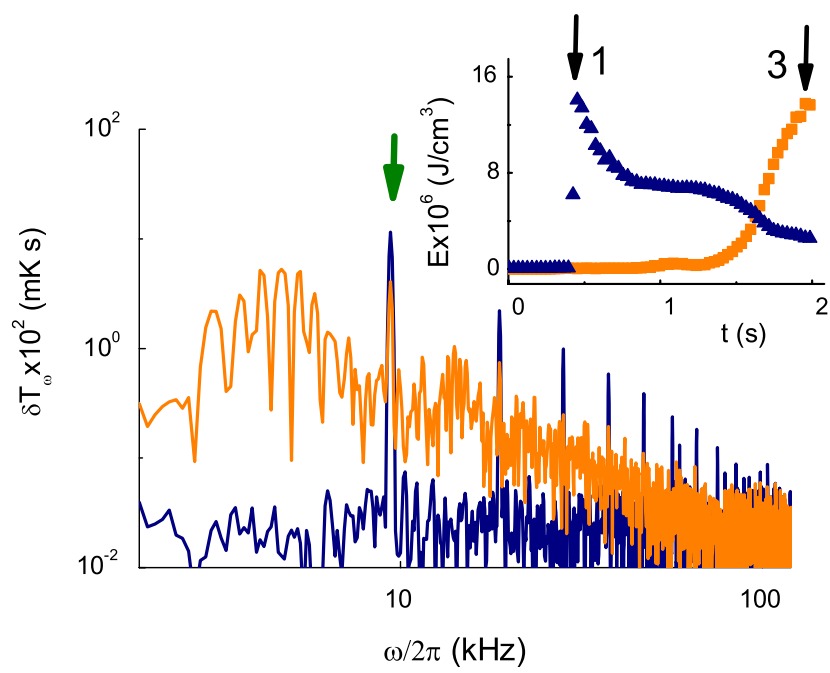

FIG. 4 (color online). Instantaneous spectra in frames 1 and 3 of Fig. 3. The lower (blue) spectrum, for frame 1, shows the direct cascade only; the upper (orange) spectrum, for frame 3, shows both the direct and inverse cascades. The (green) arrow indicates the fundamental peak at the driving frequency. Inset: Evolution of the wave energy in the low-frequency and high-frequency domains is shown by the (orange) squares and (blue) triangles respectively; (black) arrows mark the positions of frames 1 and 3 .

evolves, the rogue waves appear more frequently and, at the later stages, they merge resulting in the strong lowfrequency modulation of the signal observed in the steadystate measurements (Fig. 1).

When the subharmonics appear, a marked reduction occurs in the energy contained in the high-frequency spectral domain; see Fig. 4. A substantial proportion of the energy then flows from the driving frequency $\omega_{d}$ towards the low-frequency domain $\omega<\omega_{d}$ leading to an accumulation of wave energy there and a corresponding increase in wave amplitude. The reduction of wave amplitude seen in the high-frequency spectral domain is indicative of the onset of energy backflow towards lower frequencies, i.e., a sharing of the flux between the direct and inverse energy cascades. The decrease in energy at high frequencies within $0.397<t<1.3 \mathrm{~s}$ is attributable to relaxation processes in the direct cascade. Redistribution of wave energy due to sharing of the energy flux between the direct and inverse cascades starts at $t=1.3 \mathrm{~s}$. Note that the transient evolution shown in Figs. 3 and 4 is incomplete. The forward and inverse energy fluxes are still changing at $t=$ $2 \mathrm{~s}$, leading us to anticipate further relaxation oscillations at longer times (inaccessible with our present equipment): the transient dynamics is clearly complex, and a full characterization will require further work. Absorption of wave energy at low frequencies is probably attributable to viscous drag of the normal fluid component on the resonator walls, given that bulk second sound damping is negligibly small in this frequency range: this would be consistent with the observed strong decrease of the resonator $Q$ factor below $3 \mathrm{kHz}$. We observe hysteresis between increasing and decreasing frequency scans (bars on data points in Fig. 5). Its width, i.e., the region where the low-frequency sound waves are metastable, was less than the viscous width of the resonance.

To seek a more detailed understanding of wave energy transformation in acoustic turbulence, we used a technique [19] for direct numerical integration of the 2-fluid thermohydrodynamical equations [17], expanded up to quadratic terms in the wave amplitude. It represents the second sound waves in terms of Hamiltonian variables [7,20], as in earlier studies [8] of acoustic turbulence. Wave damping was, however, taken explicitly into account at all frequencies, a feature that is of key importance for a correct description of subharmonic generation. The main results are summarized in Fig. 5.

It is evident (inset of Fig. 5) that, for sufficiently high driving amplitude $W$, the wave develops an instability with respect to generation of low-frequency subharmonics of the driving force at $\omega_{d}$. For zero detuning from a cavity resonance, the onset of the instability occurs at a threshold $W^{*} \propto 1 / \alpha$. If the dimensionless frequency detuning $|\Delta|$ is less than a critical value $\Delta^{*} \sim 1 / Q$, the instability has a soft character, in that the amplitudes of the low-frequency

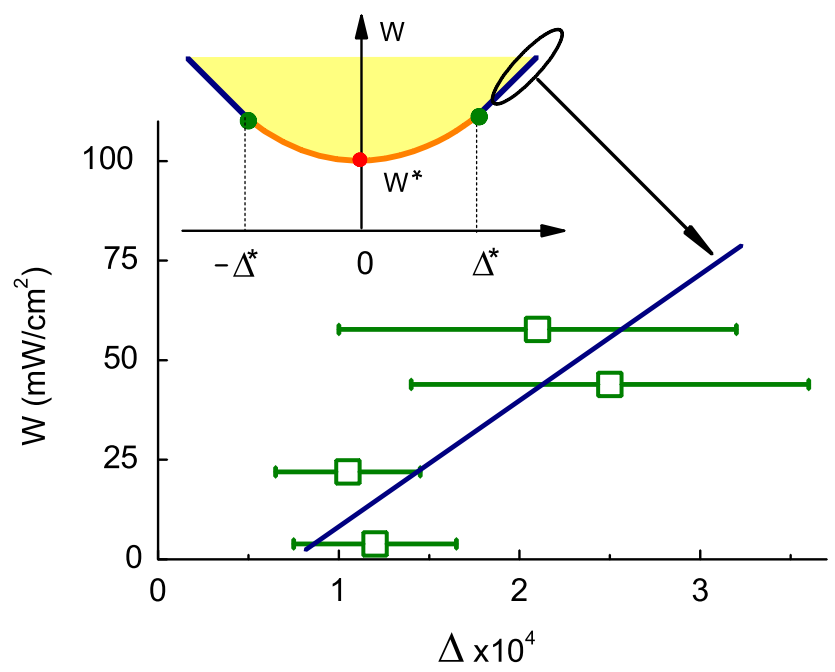

FIG. 5 (color online). Dependence of the ac heat flux density $W$ at which the instability develops on the dimensionless frequency detuning $\Delta=\left(\omega_{d}-\omega_{n}\right) / \omega_{n}$ of the driving force frequency $\omega_{d}$ from a cavity resonance $\omega_{n}$. Numerical calculations (line) are compared with measurements (points) for driving at the 96th resonance. Horizontal bars mark the widths of the hysteretic region where second sound exists in a metastable state. Inset: Bifurcation diagram showing regions of stability (unshaded) and regions of instability (yellow shaded) against the generation of subharmonics. The soft instability occurs over the (orange) line between the (green) critical points at $\pm \Delta^{*}$; outside them lies the hard instability; $W^{*}$ is the threshold value of the instability. 
waves tend to zero at the threshold bifurcation line. Outside this range, the low-frequency waves are characterized by the hard onset observed experimentally. Measurements (squares) are compared with theory (full line) in the main part of Fig. 5. The hard onset is accompanied by a finite jump in subharmonic amplitude. These two regimes of behavior are separated by critical points on the bifurcation line. The generation of subharmonics in the nonlinear oscillatory system found numerically appears to be similar to the bifurcation of an anharmonic oscillator [21]. We estimated numerically that the critical detuning parameter $\Delta^{*}$ at $T=2.08 \mathrm{~K}$ is close to $10^{-4}$, and that the critical ac heat flux density $W^{*}$ is equal to a few $\mathrm{mW} / \mathrm{cm}^{2}$, in good agreement with our observations. The experimental results shown in Fig. 1 can be understood as corresponding to the working point moving horizontally on the bifurcation diagram (Fig. 5, inset) into the (yellow) shaded region from a position just outside it, through the hard (blue) instability line. Although nonlocal (in $\omega$ space) interactions between waves at $\omega_{d}$ and low-frequency waves probably contribute to the subharmonics, due to the finite width of the lowfrequency spectral domain, our numerical estimates show that the interactions of high-frequency $\left(\omega \gg \omega_{d}\right)$ waves with subharmonics are relatively weak, consistent with our inference of two cascades.

Our experiments have revealed an inverse wave energy cascade in a turbulent acoustic system. It is responsible for a substantial increase in wave amplitude corresponding to the formation of a set of huge low-frequency subharmonics. This instability develops through formation of isolated low-frequency waves of amplitude higher than that typical of waves around them. The latter can be considered as the acoustic analogue of the giant "rogue" waves that occasionally appear on the ocean and endanger shipping. Their origin lies in the development of a decay instability of the periodic wave, i.e., a similar mechanism to that proposed [12,22] (modulation instability) to account for the creation of oceanic rogue waves [23]. Note that this mechanism differs from an alternative explanation proposed recently [24] that involves scattering of nonlinear waves on a continuous noisy background.

We acknowledge valuable discussions with A. A. Levchenko, V.E. Zakharov, E. A. Kuznetsov, V. S. L'vov, V. V. Lebedev, and T. Mizusaki. The work was supported by Engineering and Physical Sciences Research Council (U.K.), the Russian Foundation for Basic Research, Project No. 07-02-00728, and by the Presidium of the Russian Academy of Sciences under the programs "Quantum Macrophysics" and "Mathematical Methods in Nonlinear Dynamics."

*Currently at: Department of Chemical and Petroleum Engineering, Pittsburgh University, Pittsburgh, PA 15261, USA
[1] A. N. Kolmogorov, Dokl. Akad. Nauk SSSR 30, 299 (1941).

[2] V. E. Zakharov, G. Falkovich, and V.S. L'vov, Kolmogorov Spectra of Turbulence I (Springer, Berlin, 1992).

[3] S. N. Gurbatov, V. V. Kurin, L. M. Kustov, and N. V. Pronchatov-Rubtsov, Acoust. Phys. 51, 152 (2005).

[4] G. S. Bisnovatyi-Kogan and S. A. Silich, Rev. Mod. Phys. 67, 661 (1995).

[5] M. Ryutova and T. Tarbell, Phys. Rev. Lett. 90, 191101 (2003).

[6] V. S. Tsoi, Central Eur. J. Phys. 1, 72 (2003).

[7] G. V. Kolmakov, V. B. Efimov, A. N. Ganshin, P. V.E. McClintock, and L.P. Mezhov-Deglin, Phys. Rev. Lett. 97, 155301 (2006).

[8] G. Falkovich and M. Meyer, Phys. Rev. E 54, 4431 (1996).

[9] R. H. Kraichnan, Phys. Fluids 10, 1417 (1967); M. Chertkov, C. Connaughton, I. Kolokolov, and V. Lebedev, Phys. Rev. Lett. 99, 084501 (2007); J. Paret and P. Tabeling, Phys. Fluids 10, 3126 (1998); S. Nazarenko and M. Onorato, J. Low Temp. Phys. 146, 31 (2007).

[10] W. F. Vinen, M. Tsubota, and A. Mitani, Phys. Rev. Lett. 91, 135301 (2003); S. Nazarenko, JETP Lett. 84, 585 (2007).

[11] V. S. L'vov and V. B. Cherepanov, Sov. Phys. JETP 54, 746 (1981).

[12] A. I. Dyachenko and V.E. Zakharov, JETP Lett. 81, 255 (2005).

[13] P. W. Anderson and H. Suhl, Phys. Rev. 100, 1788 (1955); V.S. L'vov, Wave Turbulence under Parametric Excitation. Application to Magnetics (Springer, Berlin, 1994); T. Matsushita, R. Nomura, H. H. Hensley, H. Shiga, and T. Mizusaki, J. Low Temp. Phys. 105, 67 (1996).

[14] S. R. Spangler, J. A. Leckband, and I. H. Cairns, Phys. Plasmas 4, 846 (1997).

[15] D. Rinberg, V. Cherepanov, and V. Steinberg, Phys. Rev. Lett. 76, 2105 (1996).

[16] G. V. Kolmakov, A. A. Levchenko, M. Yu. Brazhnikov, L. P. Mezhov-Deglin, A. N. Silchenko, and P. V.E. McClintock, Phys. Rev. Lett. 93, 074501 (2004).

[17] L. D. Landau and E. M. Lifshitz, Course of Theoretical Physics, Fluid Mechanics Vol. 6 (Pergamon, New York, 1987).

[18] A. J. Dessler and W. M. Fairbank, Phys. Rev. 104, 6 (1956).

[19] A. N. Ganshin, V.B. Efimov, G. V. Kolmakov, L. P. Mezhov-Deglin, and P. V.E. McClintock, "Second sound acoustic turbulence in superfluid "He" (to be published).

[20] V. L. Pokrovskii and I. M. Khalatnikov, Sov. Phys. JETP 44, 1036 (1976).

[21] L. Mandelstam and N. Papalexi, Z. Phys. 73, 223 (1932).

[22] M. Onorato, A. R. Osborne, M. Serio, and S. Bertone, Phys. Rev. Lett. 86, 5831 (2001).

[23] R. G. Dean, in Water Wave Kinetics, edited by A. Torum and O. T. Gudmestad (Kluwer, Dordrecht, 1990), p. 609.

[24] D. R. Solli, C. Ropers, P. Koonath, and B. Jalali, Nature (London) 450, 1054 (2007). 